\title{
DIGITAL IMAGING IN ASSESSMENT OF CONSTRUCTION PROJECT PROGRESS
}

\author{
Y. Wu ${ }^{1}$ and H. Kim ${ }^{1}$ \\ ${ }^{1}$ Department of Civil and Environmental Engineering, University of Alberta, Edmonton, \\ Alberta, T6G 2G7, Canada \\ hyoungkwan@ualberta.ca
}

\begin{abstract}
This paper presents a research effort designed to produce a digital imaging-based method to efficiently assess the level of construction project progress. Cameras are widely used to monitor and record various activities on a construction site. The images produced by the cameras can be processed with digital imaging techniques to help project participants better understand the status of the project. As the first step of this on-going research, this paper focuses on an image segmentation method designed to distinguish objects of interest, such as structural members on a construction site, from other objects in the image. The segmentation method combines an edgebased segmentation method with human knowledge of the construction scene represented by image morphological operations. A promising initial research result is also presented.
\end{abstract}

Keywords: Canny Edge, Digital Imaging, Morphological Transformations, Project Control.

\section{INTRODUCTION}

A construction project is a complex development that typically occurs in an outdoor environment and involves a variety of workers, a range of equipment, and diverse materials. To successfully complete a given project, numerous business decisions must be made by all levels of management; sound decisions are based on a well-developed project scope and project plan, and, most crucially, the ongoing reassessment of the changing and dynamic condition of the construction field. However, the complex, shifting nature of construction makes it extremely challenging to assess quickly and thoroughly the status of the construction field at any particular time. For example, it is a daunting task to track the progress made across a range of physical components during the construction of a facility, let alone verify the quality of that construction on a regular basis.

Image processing has been extensively and successfully used in many sub-areas of civil engineering, such as engineering document scanning, pavement distress assessment, site evaluation via satellite imagery, studies of crack propagation and microstructure in cement-based materials, and evaluation of soil fabric, etc [1]. It is a remarkably versatile tool that provides a means to augment existing methods of analysis and also opens up a large number of possibilities for significant advances in current civil engineering practices.

Although the geotechnical and transportation engineering sub-areas of civil engineering have taken the lead in applying image processing techniques to solve practical problems over the last ten years, the advancements in hardware and software developed for digital image processing and analysis provide promising opportunities in construction management and site investigation applications. Jayaram (1990) developed a conceptual system for digital image applications to produce as-built drawings [2]. Neto et al. [3] described a method of recognizing the presence of structure components in a digital picture taken at a construction site. Abeid developed a system called PHOTO-NET to compare the last picture taken at the end of the day with the last picture taken at the end of the previous day in order to automatically record and assess the progress of different activities [3].

Image processing has great advantages and shows potential to be a powerful tool since the resultant digitized, two-dimensional array format can be processed freely to provide information about the size, shape, and location of objects that are of interest to professionals in construction management. Thus, a digital imaging-based method is proposed in this paper as an effective means to enable the automated assessment of a construction project's progress, which may lead to advanced modes of project control. During the construction of the Natural Resources Engineering Facility of the University of Alberta in Edmonton, Canada, images obtained from digital cameras installed on site were used to monitor the project; these cameras enabled the timely tracking of activities performed on site in order to study the potential capabilities, effects and limitations of image processing in construction progress control.

The first step in this research is to differentiate the structural components and equipment from the background. However, it is not a simple task to 
identify an object in a picture, especially if the picture depicts an open-field scene [3] in which the visual environment is complicated by elements such as lighting conditions, ambient facilities, weather, and other kinds of background visual interference. It is challenging to identify the various components of structures (columns, beams, and slabs, for example) and machines based on the images obtained on site. One of the most difficult problems in processing these construction-site images is that the significant amount of background visual interference is generated by various surface textures and colors, the shapes of materials and facilities, non-uniform illumination, weather conditions (i.e., snow or rain); other visibility elements also affect visual representations, such as the shooting angle and the position of the camera from which pictures are taken, or even the blockage of the line of sight.

The next important task is to accurately determine the locations or coordinates, and the size and shape parameters of the object. This task may involve multiple analyses of time-lapse images that are taken of the same object of interest at different times. Other key tasks in this research are to measure the progress made on a specific work item that is related to the identified structural components by means of timelapse pictures and to distinguish the idle and/or working equipment on site based solely on the sequential pictures. Here in this paper, we only focus on the first task: segmenting the objects of interest (the structural components and equipment), and differentiating them from the background.

\section{PROPOSED SEGMENTATION TECHNIQUES AND ALGORITHMS}

To assess construction progress by means of image processing techniques that are available, the first and most challenging step is to recognize the objects of interest, various structural components (such as the concrete columns and steel beams), and equipment on site. A robust segmentation algorithm is required because the sizes, shapes and/or locations of these objects of interest in the images change frequently as the project advances and because of the heavy background noise. Morphological transformations are the basic techniques most used to extract and alter the structure of regions in an image; these are used extensively for quantitative analysis, observation of the geometry of regions, extraction of forms for modeling and identification purposes, and so forth [5]. Human knowledge of the construction scene, such as what a concrete column should look like, can be incorporated into the segmentation method by means of the image morphological transformations. For example, an image morphological filter can be implemented to choose only those objects with vertical box shapes such as columns and excluding all other objects with different shapes. The proposed segmentation method combines such morphological filters with a Canny Edge algorithm, an edge-based segmentation method, to produce more robust segmentation results.

\subsection{Canny Edge Detector}

The Canny operator [10] was designed to be an optimal edge detector for step edges corrupted by white noise; it exploits three performance criteria covering these elements: (1) good detection (no missing edges); (2) good localization (minimal distance between true and detected edge position); and (3) one response (minimum multiple responses to single edge). It takes a grayscale image as input and produces an image that shows the positions of tracked intensity discontinuities as output. The Canny operator works in a multi-stage process. First, the input image is smoothed by Gaussian convolution (convolve the image with a Gaussian of scale sigma). Then, a simple 2-D first derivative operator is applied to the smoothed image to highlight regions of the image with high first spatial derivatives. Edges give rise to ridges in the gradient magnitude image (compute gradient magnitude and direction). The algorithm then tracks along the top of these ridges and sets zero to all pixels that are not actually on the ridge top to produce a thin line in the output (a process known as non-maximal suppression). The tracking process exhibits hysteresis that is controlled by two thresholds: high threshold $\left(T_{H}\right)$ and low threshold $\left(T_{L}\right)$ where $T_{H}>T_{L}$. Tracking can only begin at a point on a ridge higher than $T_{H}$. Then, tracking continues in both directions out from that point until the height of the ridge falls below $T_{L}$ (to eliminate spurious edges by hysteresis thresholding). This hysteresis helps to ensure that noisy edges are not broken up into multiple edge fragments.

Generally, the effect of the Canny operator is determined by three parameters: the width of the Gaussian mask used in the smoothing phase, and the upper and lower thresholds used by the tracker. Increasing the width of the Gaussian mask reduces the detector's sensitivity to noise at the expense of losing some of the finer detail in the image. The localization error in the detected edges also increases slightly as the Gaussian width is increased. Usually, the upper tracking threshold can be set quite high and the lower threshold quite low for good results. Setting the lower threshold too high will cause noisy edges to break up. Setting the upper threshold too low increases the number of spurious and undesirable edge fragments that appear in the output.

\subsection{Proposed Approach in Research}

The way to increase the possibility of delivering satisfactory segmentation results with high reliability and accuracy under the impact of heavy background 
noise is to include as much useful information as possible. As mentioned before, Canny edge detector is entirely dependent on the image gradients though it is not as strongly related to shape of objects. Based on these technical characteristics of Canny edge detector, it is possible to obtain desirable segmentation results by incorporating other image processing techniques that involve human knowledge of the construction scene.

Figure 1 shows the flowchart of the proposed segmentation method. Initially, the original RGB (Red Green Blue) color images taken on site are loaded as input data, as shown in Figure 2 (a). To save unnecessary computing time, the regions of interest are cropped in an interactive manner using functions available in MATLAB software, and then the cropped RGB image is converted into a grayscale image. After that, this grayscale image is resized using the nearest neighbor interpolation method (Figure 2 (b)). Following this step, in order to correct nonuniform illumination of the resized grayscale image, grayscale morphological opening is applied to approximate the background illumination, as shown in Figure 2 (c). Here, the background illumination is estimated through grayscale morphological opening using a disk-shaped structuring element with a radius of 15 pixels. Next, the obtained background illumination image (Figure 2 (c)) is subtracted from the resized grayscale image of interest (Figure 2 (b)). In other words, each element of the background illumination image is arithmetically subtracted from the corresponding element of the resized grayscale image. This resultant output is an enhanced grayscale image that has uniform illumination, as can be seen in Figure 2 (d).

Then, image adjustment is conducted on the enhanced grayscale image to increase visibility of objects of interest because some of the original images taken out of doors and from a far distance are not very clear. Image adjustment increases the contrast of the image by mapping the intensity values of the input image to new values, as is shown in Figure 2 (e). After the grayscale image is adjusted, median filtering is performed to simultaneously reduce noise and preserve the edges of objects; this is done using a 10-by-3 vertically rectangular neighborhood. Each output pixel contains the median value in the 10 -by-3 neighborhood around the corresponding pixel in the input image (Figure 2 (f)).

To make a further dimension of information accessible, Canny edges are subsequently detected, using a $5 \times 5$ Gaussian filter with high threshold value $T_{H}$ and low threshold value $T_{L}$, as shown in Figure 2 (g). These two threshold values, $T_{H}$ and $T_{L}$, are key parameters for segmenting the grayscale image into two regions: objects of interest and background. In order to get a desirable result, the optimal $T_{H}$ and $T_{L}$ are searched and captured in an interactive manner. The reason Canny method is applied here is that it uses two thresholds to detect strong and weak edges; it also includes the weak edges in the output only if they are connected to strong edges. The Canny method is therefore less likely than the other edge detection methods to be fooled by noise, and more likely to detect true edges.

After obtaining the desirable Canny edge binary image, the small gaps and interior holes are to be filled through the use of binary morphological closing and filling functions, successively. Morphological closing (a dilation followed by an erosion) is applied first, using a disk-shaped structure element with a radius of 5 pixels, so that the tiny gaps of the outlined Canny edges are closed, as can be seen in Figure 2 (h). Afterwards, a flood-fill operation is performed to fill holes in the Canny edge binary image, resulting in Figure 2 (i).

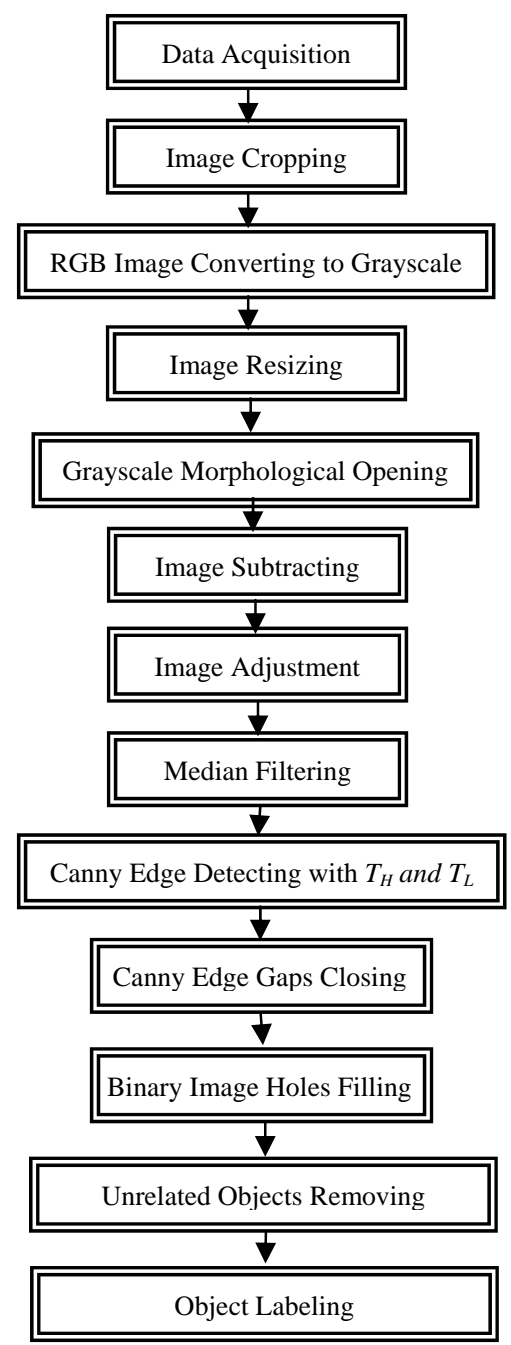

Figure 1. The Proposed Segmentation Method 


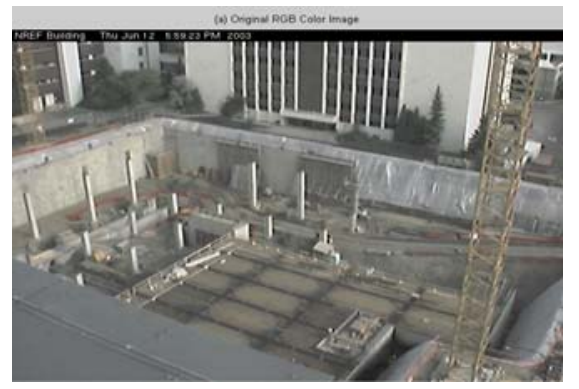

(a) Original RGB Color Image

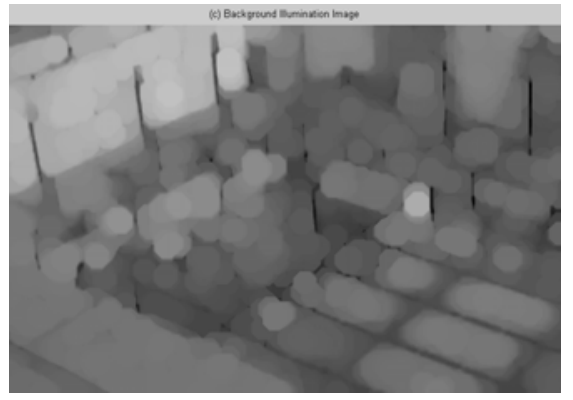

(c) Background Illumination Image

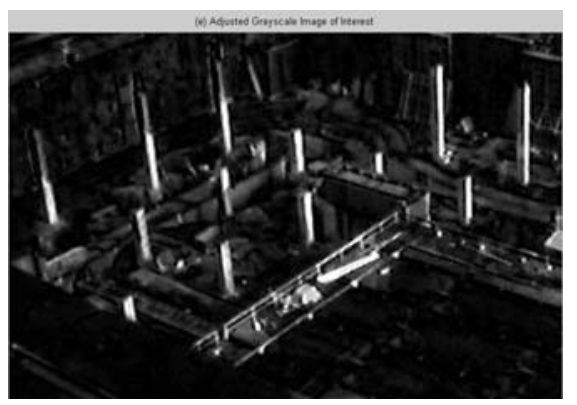

(e) Adjusted Grayscale Image

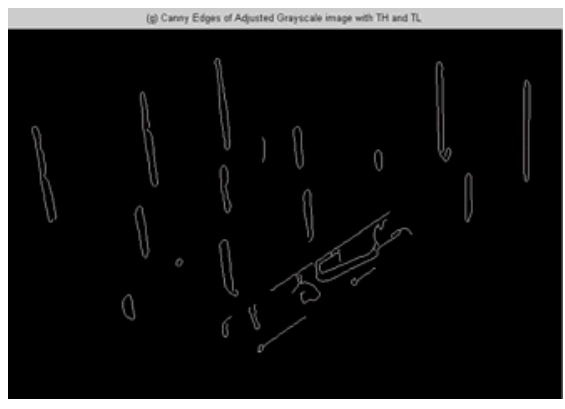

(g) Canny Edge Binary Image

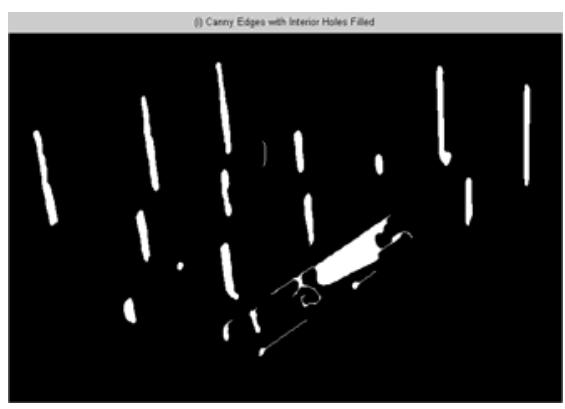

(i) Canny Edge Binary Image with Holes Filled

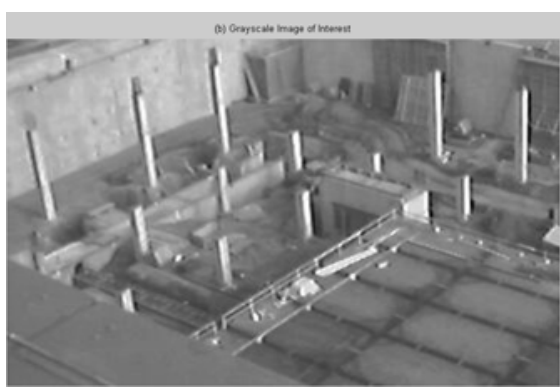

(b) Resized Grayscale Image of Interest

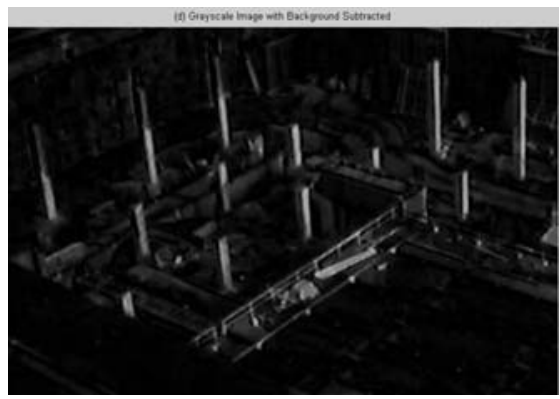

(d) Grayscale Image with Background Subtracted

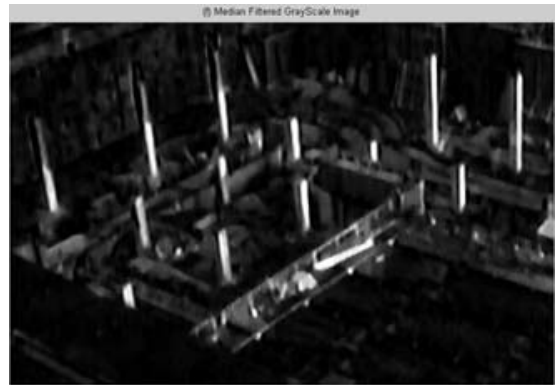

(f) Median Filtered Grayscale Image

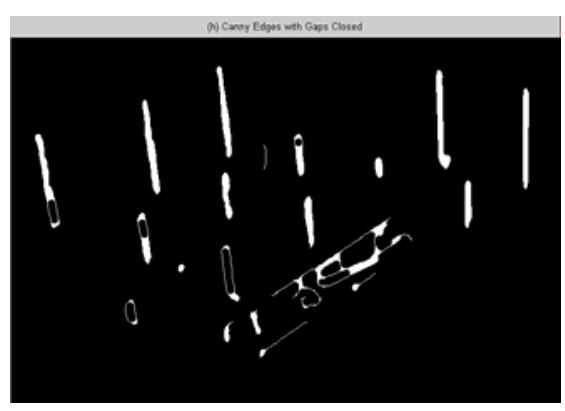

(h) Canny Edge Binary Image with Gaps Closed

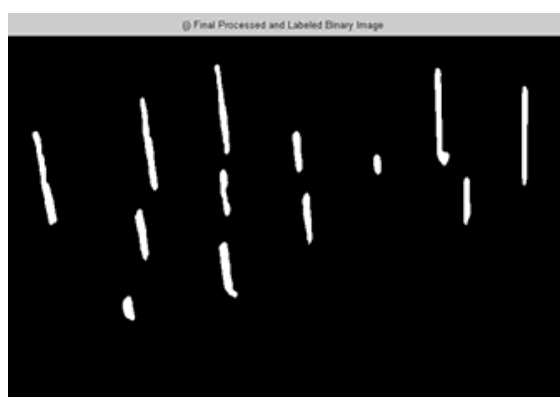

(j) Identified Regions of Interest

Figure 2. Images of Each Step of the Proposed Method 
As the program processes, some morphology functions are used to remove pixels in the binary image that do not belong to the objects of interest. The basic steps include the following: (1) Determine the connected components; (2) Compute the area of each component; and (3) Remove small objects and huge objects that are significantly beyond the area of the objects of interest. Some objects connected to the border are also removed using a morphological filter designed to exclude all the objects that touch the image border.

At the end of the operation, the objects of interest in the Canny edge binary image are labeled, as can be seen in Figure 2 (j), and properties are measured for future analysis and application in progress control. In this step, the general procedure outlined in reference [6] is used to attach a unique label (identification number) to each separate region so that every region can be identified and processed individually.

Figure 2 shows a sequence of pictures that represent the steps outlined here in order to identify reinforced concrete columns on the building construction site as an example. A comparison between Figures 2 (b) and $(\mathrm{j})$ indicates the promising result of the proposed segmentation method.

\section{DISCUSSION OF RESULTS AND FUTURE WORK}

The segmentation algorithm above successfully identified and segmented the objects of interest in the digital images taken on the construction site despite of the heavy background noise. After the objects of interest are separated, the parameters of each object in each time-lapse image can be obtained and analyzed for further studies. Since the shooting angle and position of the camera installed on site are fixed during a long period of time, major image processing parameters (for example threshold values $T_{H}$ and $T_{L}$ in Canny Edge detection) will not change sharply, and thus the data associated with objects of interest, such as the volume of concrete columns, can be automatically and efficiently obtained using the same image processing procedure aforementioned. This indicates a promising future for automatic construction progress control based on image processing techniques. To some extent, it breaks new ground to solve one of the most crucial and challenging problems in the area of construction automation.

However, further studies are needed before this method is applied successfully in more practical cases. After all, the edges detected by the algorithm discussed in this paper are not the true edges of the structural components; furthermore, some of the parameters of the 3D objects of interest are hard to obtain based solely on the 2D images. Therefore, neither the parameters of the structural components nor the productivity of each activity derived for the purpose of progress control is satisfactorily precise. To further enhance the performance of this methodology (to render it more reasonable and practical), the true edges of the objects of interest in each sample image are to be extracted and compared with the Canny edges to better investigate the relationships between them. With this in mind, advanced image processing techniques and human knowledge must be incorporated for feature extraction and comparison so that the results will be more desirable.

\section{CONCLUSIONS}

This article presents the outcome of an algorithm that can detect a structural component in an image taken of a construction site. The objective is to significantly enhance the performance of a image processing method and thus minimize the need to manually separate objects of interest in an image and, ultimately, to facilitate the dynamic progress control. The method for segmenting objects of interest is developed based on Canny Edge detector as it is incorporated with other morphological transformations. The Canny Edge detector initially outlines the objects of interest with optimal threshold values based on the enhanced grayscale image. This proposed method is effective as a means to segment structural components in digital images taken at the construction site. However, when many kinds of structural components need to be identified at the same time, or the structural components are too small (because the pictures are taken from a great distance), this algorithm needs further modification and therefore is still under development. Despite the problem mentioned above, the algorithm has produced very promising results and indicates remarkable opportunities for automatic progress control in the construction industry.

\section{REFERENCES}

[1] Lee, H. and Chou, E., "Survey of Image Processing Applications in Civil Engineering", Proceedings of Digital Image Processing: Techniques and Applications in Civil Engineering; 1st, p. 203-210, 1993.

[2] Smith, G. R. and Raynar, K., "Application of Digital Images and Processing for As-Built Construction Drawings", Proceedings of Digital Image Processing: Techniques and Applications in Civil Engineering; 1st, pp. 263-270, 1993.

[3] Neto, J. A. and Arditi, D. and Evens, M. W., "Using Colors to Detect Structural Components in 
Digital Pictures", Computer-Aided Civil and Infrastructure Engineering, Vol. 17, pp. 61-67, 2002.

[4] Kim, H., Haas, C. T., Rauch, A. F., and Browne, C., "3D Image Segmentation of Aggregates from Laser Profiling", Computer-Aided Civil and Infrastructure Engineering, Vol. 18, pp. 254-263, 2003.

[5] National Instruments Corporation, $I M A Q^{T M}$ : IMAQ Vision User Manual, 1999.

[6] Haralick, Robert M., and Linda G. Shapiro, Computer and Robot Vision, Volume I, AddisonWesley, 1992.

[7] Meyer, F. and Beucher, S., "Morphological Segmentation", Journal of Visual Communication and Image Representation, Vol. 1, pp. 21-46, 1990.

[8] The MathWorks, Image Processing Toolbox for Use with MATLAB*: User's Guide Version 4, 2003.

[9] Bleau, A. and Leon, L. J., "Watershed-Based Segmentation and Region Merging", Computer Vision and Image Understanding, Vol. 77, pp.317370, 2000.

[10] Canny, J., "Computational Approach to Edge Detection", IEEE Transactions on Pattern Analysis and Machine Intelligence, Vol. 8, No. 6, pp. 679-698, Nov 1986.

[11] Wang, W., "Binary Image Segmentation of Aggregates Based on Polygonal Approximation and Classification of Concavities", Pattern Recognition, Vol.31, No.10, pp. 1503-1524, 1998. 